\title{
Application value of ultrasound contrast in intracranial tumor surgery
}

\author{
Guoqing Sui ${ }^{\mathrm{a}}$, Dan Fei ${ }^{*}$, \\ Department of Ultrasound, the China-Japan Union Hospital of Jilin University, Changchun, China \\ a suigq15@mails.jlu.edu.cn, ${ }^{\text {b } 281740025 @ q q . c o m ~}$ \\ *Corresponding author
}

Keywords: Intracranial tumor, intraoperative ultrasonography, ultrasound contrast, operative treatment.

\begin{abstract}
Intracranial tumor is one of the most common diseases in nervous system, which has great harm to the function of human nervous system. Surgical tumor resection is the primary treatment method for the disease. Ultrasonoscopy is helpful for lesion localization and boundary determination in intracranial tumor resection surgery resection rate improvement and reduction of postoperative tumor recurrence probability. However, the deficiency of routine ultrasound examination in image contrast leads to obvious limitation thereof in clinical application. The blood perfusion difference between pathological tissue and normal tissue can be utilized to increase the image contrast in ultrasound contrast, thereby providing more accurate and clearer ultrasonic image, assisting the performer to determine tumor boundary more accurately, understand the hemoperfusion status of tumor, judge residual tumor, and improving diagnosis accuracy and surgical resection rate.
\end{abstract}

\section{Materials and methods}

Case source: 86 patients of intracranial tumor accepting ultrasound-assisted operative treatment in neurosurgery department of China-japan Friendship Hospital of Jilin University from May 2010 to March 2017 are collected.

The group is composed of 86 patients with intracranial tumor, including 52 male patients and 34 female patients. The average age of the patients is $48+-12.5$ years old, the youngest patient is 10 years old, the oldest patient is 67 years old, the median age is 50 years old. All patients were informed, and approval was obtained before surgery.

Clinical manifestations mainly include headache, limb weakness, convulsion, aphasia, etc. The course of illness ranges from two weeks to three years. Preoperative imaging shows that 64 patients have left tumors, and 22 patients have right tumors. Wherein, tumors of 23 patients are located on temporal lobes, tumors of 26 patients are located on the frontal lobe, tumors of 13 patients are located on the insular lobe, tumors of 18 patients are located on parietal lobe, and tumors of 66 patients are located on cerebellum.

\section{Instruments and drug}

Inspection equipment is Esaote mylab 90 diasonograph, which is equipped with a built-in real-time ultrasound contrast imaging technology and Qontrast ${ }^{\circledR}$ software. The mechanical index is set to 0.20 . 5-10MHZ adjustable special intraoperative porbe is configured.

SonoVue of Italy Bracco is used as ultrasound contrast agent.

\section{Inspection method}

The tumor location was determined according to preoperative MRI, CT and other image data. The craniotomy was applied to remove bone flap and expose dura mater. Routine ultrasound examination was applied for coronal, sagittal, horizontal or required planer sliding examination in bone window 
scope. The tumor lesion was displayed under the two-dimensional gray order mode. The position, morphology, boundary, internal situation and edema of surrounding brain tissue were observed. The size of the lesion and the depth to the brain surface were measured. CDFI was started to display the adjacent relationship between the tumor and the important blood vessels around it.

\section{Statistical analysis method}

SPSS13.0 software was used for statistical processing. The measurement data was expressed with mean or plus standard deviation. The t test of independent samples was applied for mean comparison. The paired t test was used for pairing, and $\mathrm{P}<0.05$ was statistically significant.

\section{Results}

The surgery process was smooth and there were no serious complications after surgery. 73 cases of tumor total resection and 13 cases of secondary tumor total resection were reviewed within one week after surgery through CT or MRI. Postoperative pathological diagnosis showed that: 70 cases with glioma were diagnosed, 20 cases had astrocytoma (WHO I - II level), 19 cases had oligodendroglioma (WHO II level), 17 cases had anaplastic astrocytoma s (WHO III level), 14 cases had glioblastoma (WHO IV level); and 16 cases ahd brain metastatic tumor.

\subsection{Tumor ultrasonic location}

All lesions were well developed by intraoperative ultrasonography. The tumors were positioned, and the positioning accuracy was 100\%. After contrast agent was injected, internal structure and boundary of the tumor were clearly displayed

\subsection{Ultrasonogram characteristics of intracranial tumor}

The normal brain tissue was hypoechoic under routine ultrasound examination. It was always enhanced after the contrast agent was injected. After all tumors were injected with contrast agent, they were enhanced at different degrees, and their enhancement time, intensity and distribution were different. Ultrasonogram accurately showed the location of tumor, the boundary of the tumor, tumor internal blood vessels, relations with adjacent blood vessels and tumor blood perfusion characteristics in real time. The time-intensity curve (TIC) was drawn through Qontrast ${ }^{\circledR}$ software, contrast enhancement policy index was obtained, and the control pathology results were shown in Table 1 in details.

Table 1 Ultrasonography of various intracranial tumors

\begin{tabular}{|c|c|c|c|c|c|c|}
\hline $\begin{array}{c}\text { Pathological } \\
\text { classification }\end{array}$ & $\begin{array}{c}\text { Case } \\
\text { number }\end{array}$ & $\begin{array}{c}\text { Greatest tumor } \\
\text { diameter }(\mathrm{cm})\end{array}$ & $\mathrm{T} 1(\mathrm{~s})$ & $\begin{array}{c}\text { Peak } \\
\text { strength } \\
(\mathrm{db})\end{array}$ & $\mathrm{T} 2(\mathrm{~s})$ & $\mathrm{T} 3(\mathrm{~s})$ \\
\hline Astrocytoma & 20 & $2.81 \pm 0.31$ & $13.0 \pm 1.5$ & $45.1 \pm 2.8$ & $29.1 \pm 2.0$ & $78.5 \pm 4.3$ \\
\hline Oligodendroglioma & 19 & $2.92 \pm 0.45$ & $9.8 \pm 2.2$ & $50.2 \pm 3.0$ & $30.0 \pm 1.8$ & $76.7 \pm 4.9$ \\
\hline $\begin{array}{c}\text { Anaplastic } \\
\text { Astrocytoma }\end{array}$ & 17 & $3.61 \pm 0.46$ & $10.2 \pm 1.4$ & $55.1 \pm 4.2$ & $28.4 \pm 2.3$ & $68.3 \pm 8.1$ \\
\hline Glioblastoma & 14 & $4.30 \pm 0.13$ & $8.8 \pm 2.1$ & $61.3 \pm 1.4$ & $26.0 \pm 1.7$ & $65.4 \pm 5.4$ \\
\hline $\begin{array}{c}\text { Brain metastatic } \\
\text { tumor }\end{array}$ & 16 & $2.32 \pm 0.51$ & $11.6 \pm 2.0$ & $53.1 \pm 3.1$ & $31.4 \pm 2.1$ & $77.3 \pm 3.2$ \\
\hline
\end{tabular}

T1: enhancement starting time; T2: time to peak; T3: enhancement extinction time

\subsection{Brain metastatic tumor}

5 cases had brain metastatic tumors. Routine ultrasound examination showed medium and high unequal echo. The lesion was surrounded by large hypoecho edema brain tissue with clear boundary. CDFI showed abundant blood flow signals around the lesion. The internal results and boundaries of 
the tumor were clearly displayed after contrast, which were manifested as irregular enhancement. The reinforcement gradually faded later.

\subsection{Residual tumor judgement}

The pathological examination results were regarded as gold standards, the comparison between routine ultrasound examination and results of pathological examination to judge neoplasm residual was shown in Table 2, judgment sensitivity was $41.7 \%$, specificity was $95.2 \%$, false positive rate was $4.8 \%$, false negative rate was $58.3 \%$; the comparison between ultrasound contrast and results of pathological examination to judge neoplasm residual was shown in Table 3, judgment sensitivity was $83.3 \%$, specificity was $100 \%$, false positive rate was 0 , and false negative rate was $16.7 \%$.

Table 2 Comparison of LGG and HGG ultrasound contrast enhancement characteristics

\begin{tabular}{|c|c|c|c|c|c|c|c|}
\hline Category & $\begin{array}{c}\text { Case } \\
\text { number }\end{array}$ & $\begin{array}{c}\text { Greatest } \\
\text { tumor } \\
\text { diameter } \\
(\mathrm{cm})\end{array}$ & $\begin{array}{c}\text { Shortest } \\
\text { distance to } \\
\text { brain surface } \\
(\mathrm{cm})\end{array}$ & T1(s) & T2(s) & T3(s) & $\begin{array}{c}\text { strength } \\
\text { (db) }\end{array}$ \\
\hline LGG & 38 & $2.91 \pm 0.59$ & $1.63 \pm 0.48$ & $12.9 \pm 1.6$ & $30.4 \pm 2.3$ & $78.7 \pm 5.7$ & $48.2 \pm 3.5$ \\
\hline HGG & 32 & $4.29 \pm 0.97$ & $1.71 \pm 0.48$ & $9.2 \pm 1.9$ & $27.2 \pm 2.5$ & $67.0 \pm 7.4$ & $60.1 \pm 3.8$ \\
\hline
\end{tabular}

LGG: low-grade gliomas; HGG: high-grade gliomas; T1: enhancement starting time; T2: time to peak; T3: enhancement extinction time

Table 3 Contrastive analysis of routine ultrasound examination and neoplasm residual evaluation with pathological examination

\begin{tabular}{|c|c|c|c|}
\hline $\begin{array}{c}\text { Routine ultrasound } \\
\text { examination }\end{array}$ & $\begin{array}{c}\text { Positive pathology } \\
\text { result }\end{array}$ & $\begin{array}{c}\text { Negative pathology } \\
\text { result }\end{array}$ & Total \\
\hline Residual & 5 & 2 & 7 \\
\hline No residual & 7 & 40 & 47 \\
\hline Total & 12 & 42 & 54 \\
\hline
\end{tabular}

\section{Discussion}

The primary treatment method for intracranial tumor is surgical resection. The basic principle of surgical resection is shown as follows: the tumor is removed to the maximum extent under the premise of protecting the nerve function ${ }^{[1]}$. Different technologies were applied in neurosurgery to reduce surgical injury in order to optimize the effect of surgical resection, such as navigation system, intraoperative MRI, intraoperative ultrasonography, etc.

Conventional gray-scale ultrasound and doppler ultrasound have low image resolution, and they have limited operation guidance functions ${ }^{[2,3]}$ aiming at intracranial lesion with deeper location and smaller diameter, especially some malignant tumors with aggressive growth characteristics. Ultrasound contrast enhances the echo comparison of blood flow and tissue through injecting ultrasound contrast agent (UCA) into the body. The blood perfusion difference between pathological tissue and normal tissue is utilized for prominently increasing the contrast of ultrasound images ${ }^{[4]}$. Tumor resection was performed smoothly for all patients in this experiment under the guidance of ultrasound contrast. The examination and implementation process of intraoperative ultrasound contrast is smooth without adverse reaction of contrast agent application. There were no serious complications after operation. It can be concluded that intraoperative ultrasound contrast is safe and feasible during application in intracranial tumor operative treatment.

It is discovered through study that the ultrasonic display rate of intracranial tumor is up to $100 \%$ because of the real-time imaging function of ultrasonoscopy ${ }^{[5]}$. However, it is more difficult for ultrasound to distinguish the lesions less than $1 \mathrm{~cm}$ in the case of insufficient experience. The discrimination ability of ultrasound is poor during judgment of malignant tumor boundary, especially 
when the tumor has been partially resected, it is difficult to accurately judge the tumor boundary ${ }^{[6,7]}$. However, the display of internal vascular structure and the relationship with adjacent blood vessels by ultrasound contrast is prominently better than routine ultrasound examination. Ultrasound contrast agent can be applied for accurately displaying the tumor location, tumor internal blood vessel and is relationship with adjacent blood vessel in real time, which is better than that before injection of contrast agent ${ }^{[8]}$.

Ultrasound contrast agent belongs to blood pool imaging agent, which is only distributed in the blood vessels and does not diffuse to other parts outside the blood vessel ${ }^{[9]}$. Therefore, The infusion sequence of contrast agent, the distribution characteristics of signals, the enhancement intensity of the lesion and other imaging characteristics can be comprehended by analyzing the relevant parameters provided by time-intensity curve (TIC) of tumor enhancement ${ }^{[10]}$. These parameters reflect the metabolic characteristics of intracranial tumor for contrast agent, which partly reflect the biological characteristics of tumors.

In the experiment, normal brain tissue always shows low enhancement, the contrast enhancement intensity of tumor tissues was significantly higher than that of surrounding brain tissue. Ultrasonography is a simple, fast and cheap real-time imaging technology. Relevant studies have shown that intraoperative ultrasonography plays certain role in detection of residual tumor. It can provide an accurate and visualized tumor edge area during the operation, thereby helping neurosurgeons to find potential residual tumor. Woydt, etc. implemented tissue biopsy in the residual cavity aiming at 45 cases of supratentorial glioma resection.

In the experiment, The residual tumors were judged according to the enhancement characteristics of tissues around the residual cavity during ultrasound contrast examination, the specificity was $100 \%$, and false positive rate was 0 . However, routine ultrasound examination residual tumor judgement sensitivity was $41.7 \%$, and false negative rate was $58.3 \%$. While the ultrasound contrast judgment sensitivity was $83.3 \%$, and the false negative rate was $16.7 \%$. The above results suggested that ultrasound contrast has higher specificity and sensitivity in the aspect of judging whether there is residual tumor or not compared with routine ultrasound examination.

\section{References}

[1] West JB, Fitzpatick JK, Toms SA, et al. Fiducial point placement and the Accuracy of point-based, rigid body registration. Neurosurgery, 2001, 48(4): 810-817.

[2] Rasmussen I, Lindseth F, Rygh O, et al. Functional neuronavigation combined with intraoperative 3D ultrasound: initial experiences during surgical resections close to eloquent brain areas and future directions in automatic brain shift compensation of preoperative data. Acta Neurochir(Wien), 2007, 149(4): 365-378.

[3] Chacko AG, Kumar NK, Chacko G, et al. Intraoperative ultrasound indetermining the extent of resection of parenchymal brain tumours-a comparative study with computed tomography and histopathology[J]. Acta Neuroehir(Wien), 2003, 145(9): 743-748.

[4] Gramiak R, Shah PM. Echocardiography of the aortic root. Invest Radiol, 1968, 3(15): 356-366.

[5] Ziskin MC, Bonakdarpour A. Contrast agents for diagnostic ultrasound. Investigative Radiology.1972, 7(6):500-512.

[6] EYang FY, Wang HE. Evaluation of the increase in permeability of the blood-brain barrier during tumor progression after pulsed focused ultrasound, International Journal of Nanomedicine, 2012, 7: 723-730.

[7] Unsgaard G, Gronningsaeter A, Ommedal S, et al. Brain operations guided by real-time two-dimensional ultrasound: new possibilities as a result of improved image quality. Neurosurgery, 2002, 51: 402-411.

[8] Y.Y.Qiu, Y.Luo, Y.L.Zhang, et al. The Correlation between Acoustic Cavitation and 
Sonoporation Involved in Ultrasound-mediated DNA Trans- fection with Polyethylenimine(PEI)In Vitro.J.of Controlled Release. 2010, 145(1):40-48.

[9] Dijkmans PA, Juffermans LJ. Microbubbles and ultrasound: from diagnosis to therapy. Eur J Echocardiogr, 2004, 5(4): 245-256.

[10] Albert FK, Forsting M, Sartor K, et al. Early postoperative magnetic resonance imaging after resection of malignant glioma: Objective evaluation of residual tumor and its influence on regrowth and prognosis. Neurosurgery, 1994, 34: 45-61. 